\title{
Spatial spectrum analysis of wave-front correction with a segmented mirror
}

\author{
Stephen Padin
}

\begin{abstract}
An expression is derived for the spatial power spectrum of wave-front errors after correction with a segmented mirror. This includes estimates of the spectral contributions of segment piston and tilt corrections and spatial aliasing by a regular array of segments. The approach allows rapid computation of wave-front error spectra in systems with highly segmented mirrors. (c) 2003 Optical Society of America

OCIS codes: $\quad 010.1080,350.1260$.
\end{abstract}

\section{Introduction}

The simplest model of a segmented mirror is a highpass filter in the spatial-frequency domain, with a sharp cutoff at $1 / 2 d$, where $d$ is the segment diameter. This type of model is common in adaptive optics (AO) system analysis. ${ }^{1-4}$ A real segmented mirror has a fairly gentle spatial-frequency cutoff, and wavefront fitting is a nonlinear process; both of these effects must be included to improve the fidelity of the mirror model. Segment adjustments can be based on wave-front measurements or on mechanical measurements of structural deformations in the telescope; but in both cases the segment array samples the measurements, and this causes aliasing. Largeamplitude, low-spatial-frequency errors, e.g., due to atmospheric phase fluctuations and gravitational and wind-induced deformations of the telescope, are aliased to high spatial frequencies and may inject significant power above the spatial bandpass of the telescope AO system. This increases the number of actuators required to achieve a particular minimum wave-front error. Aliasing of high-spatial-frequency noise down into the $\mathrm{AO}$ correction band also increases the error in the delivered wave front. This again increases the required number of actuators because atmospheric phase fluctuations and telescope deformations have lower power at high spatial frequen-

S. Padin (spadin@caltech.edu) is with the California Institute of Technology, Mail Stop 105-24, 1200 East California Boulevard, Pasadena, California 91125.

Received 16 January 2003; revised manuscript received 31 March 2003.

0003-6935/03/193721-05\$15.00/0

(C) 2003 Optical Society of America cies, so increasing the spatial bandwidth of the $\mathrm{AO}$ system decreases the aliased noise.

The focus of this paper is an estimate of the spatial spectrum of residual wave-front errors, and hence imaging performance, for a segmented mirror. This is an extension of research by Rigaut et al..$^{2}$ to include models of the spectral contributions of segment piston and tilt corrections, based on both wave-front and segment edge sensor measurements. The analysis is entirely in the spatial-frequency domain, which reduces the computation time for highly segmented mirrors because the calculations are independent of the number of segments. The approach also fits well with models of the spatial spectra of wind-induced deformations and atmospheric phase fluctuations.

\section{Wave-front Fitting in One Dimension}

The minimum rms wave-front error is obtained when segments are adjusted on the basis of a least-squares fit to measurements of the wave-front or mirror surface error. In a large, active, segmented mirror, two height sensors per segment edge are typical; 5 the sensors sample scales smaller than a segment, and the mirror surface control can approach least-squares fitting. For AO, the number of measurements and computations makes least-squares fitting impractical, so direct measurements are made of the average wave-front tilt across each segment (e.g., with a Shack-Hartmann sensor). In this section, I calculate one-dimensional (1-D) spatial spectra corresponding to wave-front corrections based on both least-squares fitting and average tilt measurements. The 1-D case is presented in some detail as an introduction to the more useful two-dimensional (2-D) analysis in Section 3. In the following, a subscript LS indicates least-squares fitting, and SH refers to a 
Shack-Hartmann (or similar average tilt) measurement.

In a least-squares fit to $N$ samples of the wave-front error, $w_{1}, w_{2} \ldots w_{N}$, taken at positions $x_{1}, x_{2} \ldots x_{N}$ across a segment, the piston is $\delta_{\mathrm{LS}}=1 / N \sum_{j=1}^{N} w_{j}$; and for $\sum_{j=1}^{N} x_{j}=0$, the tilt is $\theta_{\mathrm{LS}}=\sum_{j=1}^{N} w_{j} x_{j} / \sum_{j=1}^{N} x_{j}$ (Ref. 6). The piston measurement is equivalent to

$$
\delta_{\mathrm{LS}}(x)=\frac{1}{d} \int_{x-d / 2}^{x+d / 2} w(s) \mathrm{d} s=\frac{1}{d}[w(x) * \Pi(x / d)],
$$

where $x$ is the position on the mirror surface, $w(x)$ is the wave-front error, $\Pi(x / d)=1$ for $|x| \leq d / 2$ but 0 otherwise, and $*$ indicates convolution. The corresponding spatial spectrum is

$$
\Delta_{\mathrm{LS}}(u)=W(u) \operatorname{sinc}(\pi d u),
$$

where $u$ is the spatial frequency, and $\operatorname{sinc}(x)=(\sin$ $x) / x$. For tilt,

$$
\begin{aligned}
\theta_{\mathrm{LS}}(x) & =\frac{\int_{x-d / 2}^{x+d / 2}(s-x) w(s) \mathrm{d} s}{\int_{-d / 2}^{d / 2} s^{2} \mathrm{~d} s} \\
& =\frac{12}{d^{3}} \int_{x-d / 2}^{x+d / 2}(s-x) w(s) \mathrm{d} s \\
& =-\frac{12}{d^{3}}[w(x) * x \Pi(x / d)],
\end{aligned}
$$

and the spatial spectrum is

$$
\Theta_{\mathrm{LS}}(u)=W(u) \frac{6 i}{d}\left[\frac{\sin (\pi d u)}{(\pi d u)^{2}}-\frac{\cos (\pi d u)}{\pi d u}\right] .
$$

A Shack-Hartmann sensor measures the average of $w^{\prime}(x)$ across a segment, hence

$$
\theta_{\mathrm{SH}}(x)=\frac{1}{d}\left[w^{\prime}(x) * \Pi(x / d)\right] .
$$

Applying the Fourier-transform derivative theorem ${ }^{7}$ then yields

$$
\Theta_{\mathrm{SH}}(u)=i 2 \pi u W(u) \operatorname{sinc}(\pi d u)=W(u) \frac{2 i}{d} \sin (\pi d u) .
$$

If we transform back to the $x$ domain, $\theta_{\mathrm{SH}}(x)=(1 /$ d) $w(x) *[\mathrm{I}(x+d / 2)-\mathrm{I}(x-d / 2)]$, where $\mathrm{I}(x)$ is the impulse symbol, so the average tilt across a segment is just the gradient between two samples of the wave front taken on opposite edges of the segment. In the Shack-Hartmann case, the piston for a segment must be computed by integrating tilt across the mirror. This yields

$$
\delta_{\mathrm{SH}}(x)=\int_{0}^{x} \theta_{\mathrm{SH}}(s) \mathrm{d} s,
$$

hence

$$
\begin{aligned}
\Delta_{\mathrm{SH}}{ }^{\prime}(u) & =\Theta_{\mathrm{SH}}(u), \\
\Delta_{\mathrm{SH}}(u) & =\frac{\Theta_{\mathrm{SH}}(u)}{i 2 \pi u}=W(u) \operatorname{sinc}(\pi d u),
\end{aligned}
$$

which is the same as for a least-squares fit to the wave front. In general, we can write $\Delta(u)=p(u) W(u)$, and $\Theta(u)=t(u) W(u)$, where $p(u)$ and $t(u)$ are the measurement functions for segment piston and tilt.

To correct wave-front errors, we adjust the segments based on the values of $\delta(x)$ and $\theta(x)$ at the segment centers. This is equivalent to sampling $\delta(x)$ and $\theta(x)$ with $\operatorname{III}(x / d)$ (an infinite array of impulse symbols with spacing $d$ ) and then convolving with $-\Pi(x / d)$ and $-x \Pi(x / d)$ to generate the piston and tilt components $\delta_{M}(x)$ and $\theta_{M}(x)$ corresponding to the mirror surface (see Fig. 1). For piston,

$$
\delta_{M}(x)=-[\delta(x) \operatorname{III}(x / d)] * \Pi(x / d),
$$

hence

$$
\Delta_{M}(u)=-[\Delta(u) * \operatorname{III}(u d)] \operatorname{sinc}(\pi d u) ;
$$

and for tilt,

$$
\theta_{M}(x)=-[\theta(x) \operatorname{III}(x / d)] * x \Pi(x / d),
$$

so

$$
\begin{aligned}
\Theta_{M}(u)= & \frac{i d}{2}[\Theta(u) * \operatorname{III}(u d)] \\
& \times\left[\frac{\sin (\pi d u)}{(\pi d u)^{2}}-\frac{\cos (\pi d u)}{\pi d u}\right] .
\end{aligned}
$$

In general, $\Delta_{M}(u)=[p(u) W(u) * \operatorname{III}(u d)] P(u)$, and $\Theta_{M}(u)=[t(u) W(u) * \operatorname{III}(u d)] T(u)$, where $P(u)$ and $T(u)$ are the correction functions for segment piston and tilt.

The corrected wave front is the sum of $w(x)$ and the piston and tilt components corresponding to the mirror surface. If $w(x)$ is a realization of a random stationary process, components aliased from different spatial frequencies are uncorrelated. The statistical spatial power spectrum of residual wave-front errors, after correction for segment pistons and tilts, is then

$$
\begin{aligned}
\Phi(u)= & |W(u)|^{2}|1+p(u) P(u)+t(u) T(u)|^{2} \\
& +\sum_{j \neq 0}\left|W\left(u+\frac{j}{d}\right)\right|^{2} \mid p\left(u+\frac{j}{d}\right) P(u) \\
& +\left.t\left(u+\frac{j}{d}\right) T(u)\right|^{2},
\end{aligned}
$$

where the measurement and correction functions are given in Table 1. The first term in Eq. (14) is the square of the corrected wave-front amplitude in the 
Wave-front error

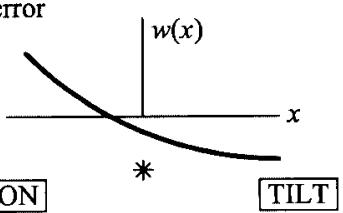

Measurement function

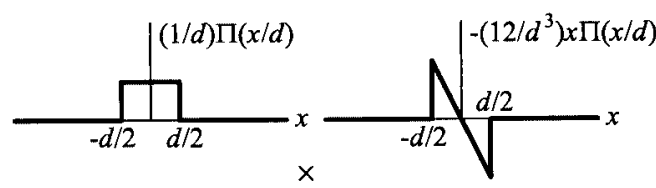

Sampling function

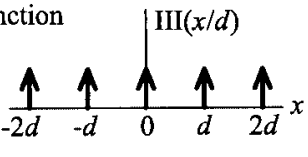

Measured wave front $\|$
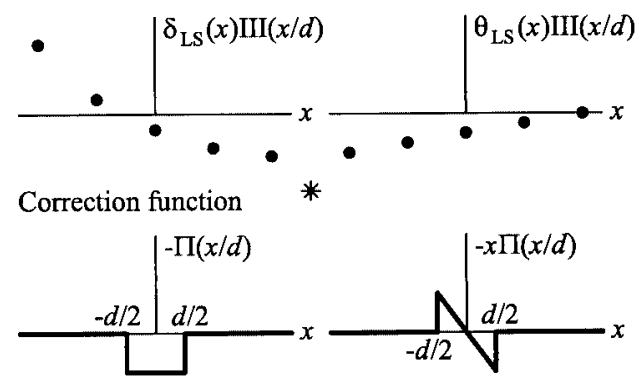

Mirror surface

॥

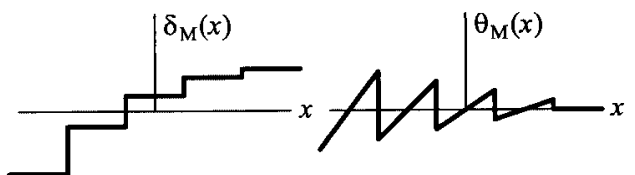

Fig. 1. Mirror surface profile calculation for wave-front error correction in one dimension, with segment tilts from a least-squares fit to the wave front. The operations run from top to bottom; $\times$ and $*$ represent multiplication and convolution, and $\uparrow$ is an impulse symbol. Applying the measurement and sampling functions yields the wave-front piston and tilt at each segment, and the correction functions generate the corresponding mirror surface. The residual wave-front error is $w(x)+\delta_{M}(x)+\theta_{M}(x)$.

unaliased $(j=0)$ component; the $j$ th term in the summation is the power aliased from spatial frequency $u+j / d$. A similar analysis applies to an $\mathrm{AO}$ system with a continuous face-sheet deformable mirror, but in this case $d$ is the actuator spacing. ${ }^{2}$ If extending a single actuator a distance $z$ causes adjacent actuators to move a distance $c z$, the actuator spacing in the correction functions can be increased by a factor of $1+c$ to roughly model the interactuator coupling.

\section{Wave-Front Fitting in Two Dimensions}

For a 2-D wave-front error, $w(\mathbf{x})=w(x, y)$, we must include $x$ and $y$ components of the tilt, so Eq. (14) becomes

$$
\begin{aligned}
\Phi(\mathbf{u})= & |W(\mathbf{u})|^{2} \mid 1+p(\mathbf{u}) P(\mathbf{u})+t_{x}(\mathbf{u}) T_{x}(\mathbf{u}) \\
& +\left.t_{y}(\mathbf{u}) T_{y}(\mathbf{u})\right|^{2} \\
& +\sum_{\substack{j, k \\
\xi \neq 0}}\left|W\left(\mathbf{u}+\boldsymbol{\xi}_{j, k}\right)\right|^{2} \mid p\left(\mathbf{u}+\boldsymbol{\xi}_{j, k}\right) P(\mathbf{u}) \\
& +t_{x}\left(\mathbf{u}+\boldsymbol{\xi}_{j, k}\right) T_{x}(\mathbf{u})+\left.t_{y}\left(\mathbf{u}+\boldsymbol{\xi}_{j, k}\right) T_{y}(\mathbf{u})\right|^{2},
\end{aligned}
$$

where $\mathbf{u}=(u, v)$ is the 2-D spatial frequency, and $\boldsymbol{\xi}_{j, k}$ is a point (e.g., at column $j$, row $k$ ) in the segment sampling function. The power spectrum of wavefront errors as a function of radius in the $(u, v)$ plane is a more useful parameter for plots, and the normalized form of this is

$$
\Psi(|\mathbf{u}|)=\frac{1}{2 \pi|W(0)|^{2}} \int_{-\pi}^{\pi} \Phi(|\mathbf{u}|, \phi) \mathrm{d} \phi,
$$

where $\phi=\arg (\mathbf{u})$.

For square segments, the measurement and correction functions are separable products (see Table 2 ), and the segment sampling function is simply $\operatorname{III}(u d) \operatorname{III}(v d)$, so Eq. (15) can be evaluated easily. Figure 2 shows predicted wave-front error spectra, along with results from a computer simulation, for an array of square segments. In this case, the uncorrected wave-front error spectrum is flat, with a spatial bandwidth $1 / d$, and all the power at $|\mathbf{u}|>$ $1 / d$ was aliased from lower spatial frequencies. Least-squares and average tilt corrections give similar total aliased power, but the spectra differ in detail. For the simulation, I started with a test wave front consisting of random numbers on a square grid with $2^{10} \times 2^{10}$ points, assigned to $2^{7} \times$ $2^{7}$ segments, with $2^{3} \times 2^{3}$ points per segment. This gives a wide enough range of spatial frequencies for a reasonable test of Eq. (15), but the computation time is not excessive $(\sim 10 \mathrm{~min}$ on an 800 $\mathrm{MHz}$ personal computer). The spatial spectrum of the test wave front, calculated by means of a fast Fourier transform, was truncated to limit the spatial bandwidth and transformed back to the wavefront domain. Then I removed wave-front pistons

Table 1. Measurement and Correction Functions in One Dimension

\begin{tabular}{lll}
\hline Error & \multicolumn{1}{c}{ Measurement Function } & Correction Function \\
\hline Piston & $p(u)=\operatorname{sinc}(\pi d u)$ & $P(u)=-\operatorname{sinc}(\pi d u)$ \\
Tilt & $t_{\mathrm{LS}}(u)=\frac{6 i}{d}\left[\frac{\sin (\pi d u)}{(\pi d u)^{2}}-\frac{\cos (\pi d u)}{\pi d u}\right]$ & $T(u)=\frac{i d}{2}\left[\frac{\sin (\pi d u)}{(\pi d u)^{2}}-\frac{\cos (\pi d u)}{\pi d u}\right]$ \\
& $t_{\mathrm{SH}}(u)=\frac{2 i}{d} \sin (\pi d u)$ & \\
\hline
\end{tabular}




\begin{tabular}{llc}
\hline Error & \multicolumn{1}{c}{ Measurement Function } & Correction Function \\
\hline Piston & $p(\mathbf{u})=\operatorname{sinc}(\pi d u) \operatorname{sinc}(\pi d v)$ & $P(\mathbf{u})=-\operatorname{sinc}(\pi d u) \operatorname{sinc}(\pi d v)$ \\
$x$ tilt & $t_{x \mathrm{LS}}(\mathbf{u})=\frac{6 i}{d}\left[\frac{\sin (\pi d u)}{(\pi d u)^{2}}-\frac{\cos (\pi d u)}{\pi d u}\right] \operatorname{sinc}(\pi d v)$ & $T_{x}(\mathbf{u})=\frac{i d}{2}\left[\frac{\sin (\pi d u)}{(\pi d u)^{2}}-\frac{\cos (\pi d u)}{\pi d u}\right] \operatorname{sinc}(\pi d v)$ \\
$t_{x \mathrm{SH}}(\mathbf{u})=\frac{2 i}{d} \sin (\pi d u) \operatorname{sinc}(\pi d v)$ & $T_{y}(\mathbf{u})=\frac{i d}{2}\left[\frac{\sin (\pi d v)}{(\pi d v)^{2}}-\frac{\cos (\pi d v)}{\pi d v}\right] \operatorname{sinc}(\pi d u)$ \\
$t_{y \mathrm{LS}}(\mathbf{u})=\frac{6 i}{d}\left[\frac{\sin (\pi d v)}{(\pi d v)^{2}}-\frac{\cos (\pi d v)}{\pi d v}\right] \operatorname{sinc}(\pi d u)$ & \\
$t_{y \mathrm{SH}}(\mathbf{u})=\frac{2 i}{d} \sin (\pi d v) \operatorname{sinc}(\pi d u)$ & \\
\hline
\end{tabular}

and tilts to simulate the segment control and calculated the spatial spectrum of the corrected wave front. Gridding the test wave front causes aliasing, which has little effect at low spatial frequencies, but roughly doubles the power at $|\mathbf{u}|=a /(2 d)$, where $a$ is the number of points across a segment.
A crude, but easy to compute, estimate of the aliasing is included in Fig. 2 where the predicted spectra are scaled for $|\mathbf{u}|>1 / d$ by a factor of $(2 d|\mathbf{u}|+a-$ $4) /(a-2)$. Errors in this estimate are responsible for the slightly higher power for the simulated versus predicted results at high spatial frequencies.
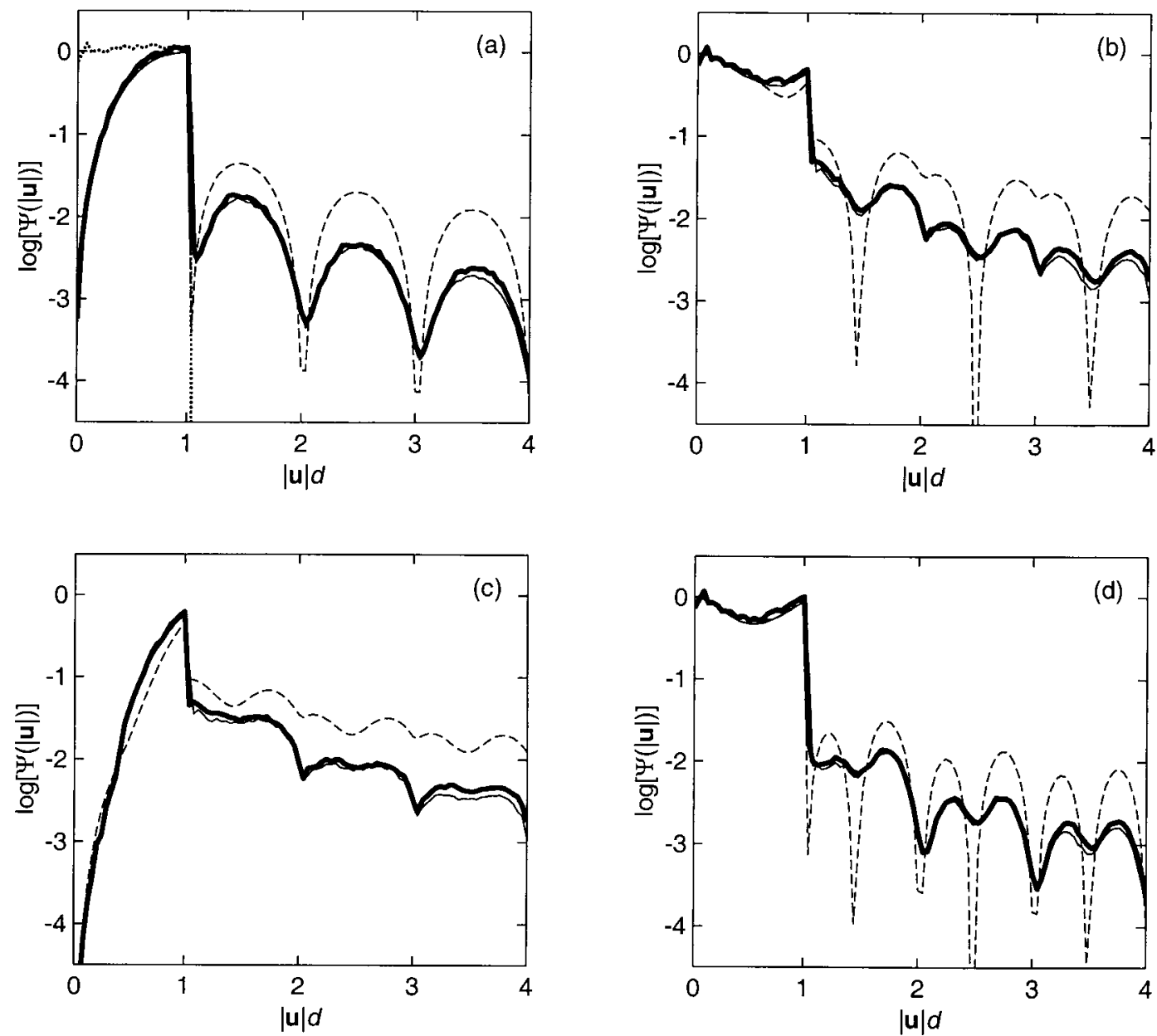

Fig. 2. Normalized wave-front error spectra for an array of square segments, after correction for (a) segment pistons, (b) tilts calculated by least-squares wave-front fitting, (c) pistons and least-squares tilts together, and (d) tilts measured by a Shack-Hartmann sensor. In each plot, the bold solid curve is a simulation of $2^{7} \times 2^{7}$ segments, with $2^{3} \times 2^{3}$ wave-front samples per segment. The thin solid curve (which is mostly hidden by the bold curve) is the spectrum predicted by Eqs. (15) and (16), with measurement and correction functions from Table 2 and $\xi_{j, k}=(j / d, k / d)$. The dashed curve is the 1-D spectrum predicted by Eq. (14), and the dotted curve in (a) is the normalized uncorrected wave-front error spectrum. 


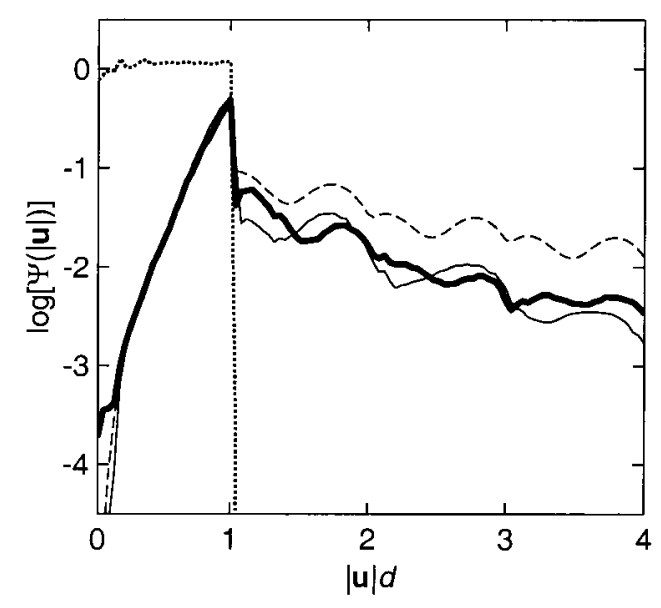

Fig. 3. Normalized wave-front error spectra for an array of hexagonal segments. The bold solid curve is a simulation of piston and least-squares tilt correction for $2^{7} \times 2^{7}$ segments, with 56 wave-front samples per segment. The thin solid curve is the spectrum predicted by Eqs. (15) and (16), with piston functions $p(\mathbf{u})=$ $-P(\mathbf{u})=2\left[J_{1}(s)\right] / s$, where $\left.s=\pi d|\mathbf{u}| 1.22 \cos (\pi / 6)\right]$, tilt functions from Table 2, and $\xi_{j, k}=\left(\left\{j-\left[(-1)^{k}-1\right] / 4\right\} / d \cos (\pi / 6), k / d\right)$. The dashed curve is the 1-D spectrum predicted by Eq. (14), and the dotted curve is the normalized uncorrected wave-front error spectrum. Predicted spectra include an estimate of the aliasing that is due to the gridding of the wave front in the simulation.

Real segmented mirrors are made of hexagons, and the measurement and correction functions are complicated. ${ }^{8}$ However, a useful estimate of the wave-front error can be obtained with circular-segment piston functions and square-segment tilt functions, as in Fig. 3 . In this case, the segment sampling function is a hexagonal lattice of impulse symbols, with spacing $1 /[d \cos (\pi / 6)]$, where $d$ is the distance across the segment flats. The circular-segment piston functions are scaled so that the first zero is at the lattice spacing,

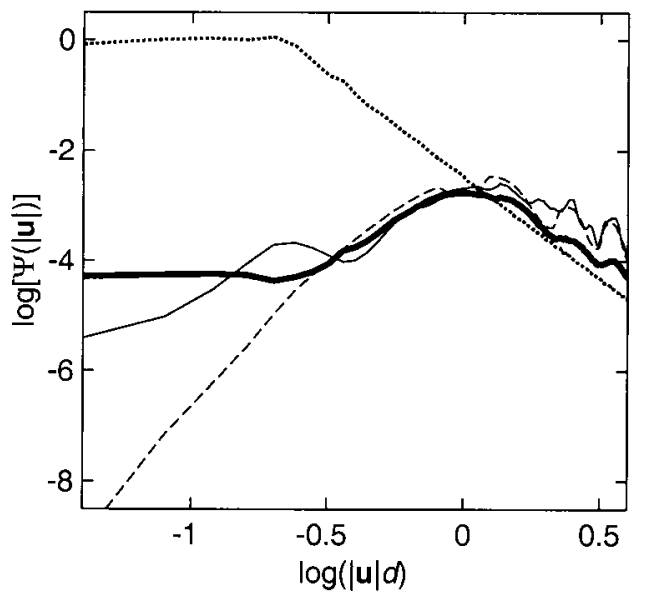

Fig. 4. Same as Fig. 3, but for an uncorrected wave-front error spectrum $\Psi\left(|\mathbf{u}| \leq 1 / L_{0}\right)=1$ and $\Psi\left(|\mathbf{u}|>1 / L_{0}\right)=\left(|\mathbf{u}| L_{0}\right)^{-11 / 3}$, with $L_{0}=5 d$. This represents atmospheric phase fluctuations for Kolmogorov turbulence ${ }^{9}$ with an outer scale of five segment diameters. which is roughly equivalent to matching the areas of the circular and hexagonal segments. The simple model gives a reasonable estimate of the aliased power, but it does not accurately predict spectral features. Figure 4 shows a similar calculation, but for an uncorrected wave-front error spectrum corresponding to atmospheric phase fluctuations. The excess noise in the corrected spectrum at $|\mathbf{u}|>1 / d$ was aliased from low spatial frequencies. Aliasing also occurred from high to low spatial frequencies, but this is masked by the decrease in power for $|\mathbf{u}|<1 / d$. In Figs. 3 and 4 , differences between the 2-D models and simulations are due to the choice of only approximate piston and tilt functions, except at low spatial frequencies where the simulations have excess power as a result of truncating the segment array.

\section{Conclusion}

The 2-D wave-front correction model of Eq. (15) allows rapid computation of the spatial spectrum of wavefront errors in a system with a segmented deformable mirror. This spatial-frequency approach is particularly attractive for mirrors with many segments because the computations are much simpler than a full simulation of the wave front. Hexagonal segments are difficult to model because the Fourier transform of a hexagon cannot be expressed as a single function, but a useful estimate of the wave-front error can be obtained with circular-segment piston functions and square-segment tilt functions; even a simple 1-D model can predict the wave-front error within a factor of a few over a wide range of spatial frequencies.

This research was supported by the Caltech Discovery Fund.

\section{References}

1. J. W. Hardy, Adaptive Optics for Astronomical Telescopes (Oxford U. Press, Oxford, UK, 1998), p. 196.

2. F. Rigaut, J. Veran, and O. Lai, "An analytical model for ShackHartmann-based adaptive optics systems," in Adaptive Optical System Technologies, D. Bonaccini and R. K. Tyson, eds., Proc. SPIE 3353, 1038-1048 (1998).

3. J. M. Beckers, "Adaptive optics for astronomy," Annu. Rev. Astron. Astrophys. 31, 13-62 (1993).

4. D. G. Sandler, S. Stahl, J. R. P. Angel, M. Lloyd-Hart, and D. McCarthy, "Adaptive optics for diffraction-limited infrared imaging with 8-m telescopes," J. Opt. Soc. Am. A 11, 925-945 (1994).

5. J. E. Nelson, T. S. Mast, and S. M. Faber, eds., "The design of the Keck Observatory and Telescope," Keck Observatory Rep. 90 (Keck Observatory, Kamuela, Hawaii, 1985).

6. P. R. Bevington, Data Reduction and Error Analysis for the Physical Sciences (McGraw-Hill, New York, 1969), p. 103.

7. R. N. Bracewell, The Fourier Transform and its Applications (McGraw-Hill, New York, 1986), p. 117.

8. G. Chanan and M. Troy, "Strehl ratio and modulation transfer function for segmented mirror telescopes as functions of segment phase error," Appl. Opt. 38, 6642-6647 (1999).

9. V. I. Tatarskii, The Effects of the Turbulent Atmosphere on Wave Propagation (National Technical Information Service, U.S. Department of Commerce, Springfield, Va., 1971), Chap. 1B. 